DOI: $10.3901 / J M E .2019 .09 .080$

\title{
考虑多尺度接触状态的新接触模型
}

\author{
运賁德 ${ }^{1}$ 丁 北 $^{2}$ \\ (1. 北京航空航天大学能源与动力工程学院 北京 100083 ; \\ 2. 中国民航大学机场学院 天津 300300)
}

\begin{abstract}
摘要: 提出了一种接触模型, 模型的建立基于经典的 Hertz 接触力学理论和现有的分形接触模型。研究了单个微凸体接触状 态随尺度序数 $n$ 的变化规律, 考虑了不同尺度序数 $n$ 的影响而引入线性因子对模型进行修正, 采用了与以往分布函数计算方 法不同的思路, 在此基础上对微凸体面积分布函数积分求和, 获得了接触表面真实接触载荷与接触面积的关系。结果表明: 单个微凸体的临界参数对接触参数有影响; 微凸体的变形顺序为弹性, 弹塑性, 塑性变形, 与传统的接触模型一致; 线性因 子与特征系数 $G$, 分形维数 $D$ 有一定的关系; 新的分布函数和线性因子的引入提高了接触参数的精度; 考虑多尺度特性的新 模型相比经典的 $\mathrm{GW}$ 模型, MB 模型与 Bhushan 试验数据更接近, 尤其在接触载荷较大时; 与现有的考虑尺度因素的接触模 型做比较, 计算精度有了更进一步的提高。
\end{abstract}

关键词: 粗精表面; 微凸体; 弹塑性; 多尺度; 接触模型

中图分类号: TH117

\section{New Fractal Contact Model Considered Multi-scale Levels}

\author{
YUN Ruide ${ }^{1}$ DING Bei ${ }^{2}$
}

(1. School of Energy and Power Engineering, Beihang University, Beijing 100083;

2. School of Airport, Civil Aviation University of China, Tianjin 300300)

\begin{abstract}
A new elastic-plastic model for contact of rough surfaces is presented. The model based on the traditional contact theory of Hertz and the presented fractal contact models is developed. Research the regularity of the contact condition of micro-bulge, considered on the multi contact asperity in different fractal parameters in the first detail. Then, to modify the contact areas by considering the multi-scale levels and a new way use to calculate the distribution function. The model developed evaluates that the multi-scale parameter will influence the values of critical contact parameters, the deformation of contact area will be the conditions of elastic, elastic-plastic and plastic successively and the effect factor has relationship with $G$ and $D$. Besides, by considering the effect factor and using new distribution function, the new model gains the more precise results than the model of GW and MB. It is show that the predict values from present model is fitting closer than the values from MB model and GW model to the results of Bhushan' experimental observation. The comparison results strongly indicate that the new model is more reasonable in describing the contact behavior between rough surfaces.
\end{abstract}

Key words: rough surfaces; asperity; elastic-plastic; multi-scale levels; contact model

\section{0 前言}

接触表面的真实接触性态对工程应用中的摩擦 磨损 ${ }^{[1-2]}$ 、润滑密封 ${ }^{[3]}$ 、接触刚度 ${ }^{[4]}$ 等工程参数有重 要的影响。表面微凸体看似非常的平整光滑, 但是 通过对其局部放大后会发现, 其接触表面是由一系 列的微凸体构成, 实际接触面仅仅发生在一部分微 凸体表面。因此, 研究接触面的真实接触面积和载

20180617 收到初稿, 20181204 收到修改稿
荷就显得尤为重要。

1966 年, GREENWOOD 和 WILLIAMSON ${ }^{[5]}$ 发现许多工程表面上的凸峰高度近似于高斯分布, 并基于三个基本假设提出了著名的 $\mathrm{GW}$ 模型, 为粗 粮表面间的接触理论奠定了基础。GW 模型虽然对 粗楉表面进行简化计算, 但与真实接触参数误差较 大。为此, 在将近 30 年的时间里, 许多学者对 GW 模型进行了许多方面的修正。如 WHITEHOUSE 和 ARCHARD 等 ${ }^{[6]}$ 舍弃了微凸体半径相同的假设, 认 为高的微凸体具有高的峰顶; NAYAK ${ }^{[7]}$ 引入了广义 联合概率密度函数, 应用在二维和三维问题中; 
BUSH 等 ${ }^{[8]}$ 考虑了微凸体在 $X$ 方向和 $Y$ 方向曲率变 化的影响; CIAVARELLA 等 ${ }^{[9]}$ 考虑了微凸体之间的 相互影响; ZHAO 等 ${ }^{[10-13]}$ 提出了弹塑性接触的影响。 统计学模型给出了小载荷下的接触参数求解方法, 但是其计算结果受制于设备的精度和采样长度等因 素的影响, 在大载荷下计算结果偏差较大。基于统 计方法建立的模型不能对粗糙面有一个定量的分 析, 从而结果缺少一定的客观性。

基于此, 1991 年 MAJUMDAR 和 BHUSHAN 提出了以分形几何为基础的接触面分形模型, $M B$ 模型 ${ }^{[14]}$ 。MB 模型在建立时仅仅考虑了弹性和塑性 接触状态, 而忽略了中间的弹塑性过渡状态; KOGUT 和 $\mathrm{ETSION}^{[15]}$ 在此基础上应用有限元理论 建立了弹塑性阶段的接触规律。后来建立的很多分 形模型都只是考虑了单一尺度下的接触状态, 而忽 略了尺度对接触状态的影响; 于是, MORAG 等 ${ }^{[16]}$ 基于分形模型和 Hertz 接触理论证明了临界参数与 尺度相关; JACKSON 等 ${ }^{[17]}$ 提出了一种基于微凸体 的多尺度模型, 假设小尺寸微凸体分布在大尺寸微 凸体上; WRIGGERS 等 ${ }^{[18]}$ 运用有限元理论对接触 面各个尺度接触参数进行分析; 孙伟等 ${ }^{[19]}$ 应用小波 理论分解真实接触面的试验数据, 再应用有限元理 论建立宏-微观尺度的接触刚度模型。史建成等 ${ }^{[20]}$ 通过有限元和小波分解理论对 8 个不同层级下的接 触参数进行了研究。缪小梅等 ${ }^{[21]}$ 在 MORAG 和 ETSION 的基础上研究了单个微凸体临界接触参数 随尺度的变化关系。丁雪兴等 ${ }^{[22]}$ 基于特定尺度参数 下, 研究了分形参数与各接触参数的变化关系。原 圆等 ${ }^{[23-24]}$ 研究了单个微凸体临界接触参数与尺度序 数 $n$ 的关系。但是以上研究对多尺度序数 $n$ 在接触 参数中的考虑仍有提升的空间。

基于上述研究, 本文对表面多尺度序数 $n$ 进行 考虑, 引入线性因子和新的分布函数进行求解, 建 立了一种新的计算模型。通过与经典模型对比, 验 证新模型具有较高的精度。

\section{1 几何模型的建立}

粗䊁表面具有自仿射与多尺度特性, Majumdar 等 ${ }^{[14]}$ 用 W-M 分形函数来近似表征粗糙表面 2 维轮 廓曲线，表达式如下

$$
z(x)=G^{D-1} \sum_{n_{\min }}^{\infty} \frac{\cos \left(2 \pi \gamma^{n} x\right)}{\gamma^{(2-D) n}} \quad 1<D<2
$$

式中, $z(x)$ 表示粗䊁表面轮廓曲线的高度; $x$ 为轮廓 位移坐标; $D$ 为表面轮廓分形维数; $G$ 为轮廓特征
系数; $\gamma$ 为大于 1 的常数, 取 $\gamma=1.5^{[14,16],} \gamma^{n}$ 表示 随机轮廓的空间频率, 决定表面粗粘度的频谱; $n_{\text {min }}$ 为与轮廓结构对应的最低截止频率指数。轮廓曲线 由 $D, G, n$ 决定, 最低截止频率和取样长度有关, $D$ 和 $G$ 可由二维 W-M 函数的功率谱获得。如图 1 所示, 在不同放大倍数下, W-M 函数的外轮廓曲线 具有相似性。

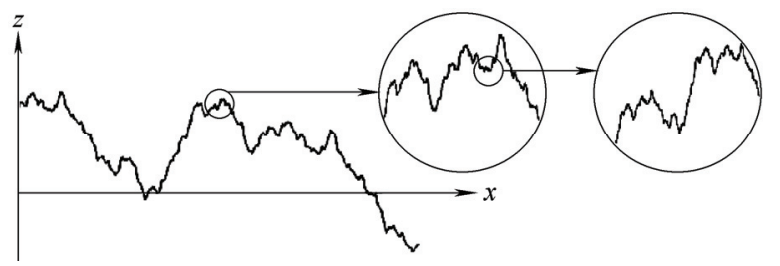

图 1 分形函数的轮廓曲线

\section{2 表面接触模型}

两粗粘表面间的接触可以等效为一个刚性光滑 平面和一个粗粘面的接触, 且满足如下假设条件: (1) 只考虑微凸体各向同性的分形特征; (2) 忽略接 触过程中微凸体间的相互作用; (3) 微凸体发生形变 时不考虑变形引起的材料硬化效应; (4) 不考虑摩擦 力的影响, 只考虑法向接触效应。

\section{1 单个微凸体模型的建立}

由 W-M 分形函数可知, 粗糙表面是由不同尺 度序数下的轮廓线 $n$ 所叠加而成的，对于任意尺度 序数 $n$ 对应下的 $L=1 / \gamma^{n}$ ，其轮廓曲线为

$$
z(x)=G^{D-1} \cdot L^{2-D} \cdot \cos \left(\frac{\pi x}{L}\right) \quad-\frac{L}{2}<x<\frac{L}{2}
$$

单个微凸体的变形示意图如图 2 所示, 其中 $L$ 为微凸体的尺寸直径; $\delta$ 为微凸体的幅值高度; $\omega$ 为 刚性平面对微凸体施加的变形量, 其值的大小满足 $0<\omega \leqslant \delta ; d$ 代表了刚性平面与接触面的距离, 其 值的大小由文献[14]推出。图 2 中点画线部分代表 了微凸体的真实轮廓形貌, 而实线部分则为微凸体 变形后形貌特征。

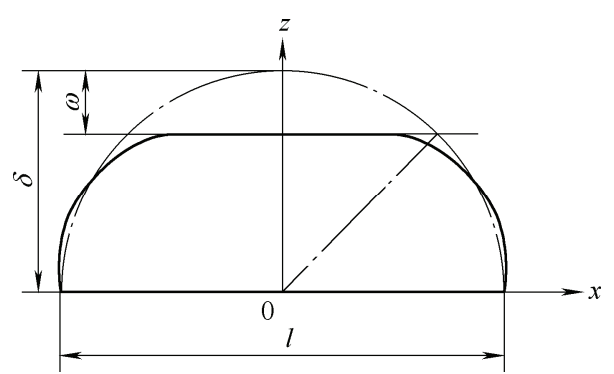

图 2 微凸体接触模型示意图 
由式(2)得微凸体表面曲率半径 $R$ 为

$$
R=\frac{1}{\left|\mathrm{~d}^{2} z / \mathrm{d} x^{2}\right|_{x=0}}=\frac{L^{D}}{\pi^{2} G^{D-1}}
$$

由式(2)得微凸体的高度 $\delta$ 为

$$
\delta=z(0)=G^{D-1} \cdot L^{2-D}
$$

微凸体的实际变形量为

$$
\omega=\delta-d
$$

两粗精面的接触, 随着接触力的逐渐增大, $d$ 逐渐减小, $\omega$ 逐渐增加, 由经典 Hertz 接触理论 ${ }^{[25]}$ 和 Kogut-Etsion 接触理论 ${ }^{[15]}$ 可知: 微凸体的变形分 为 4 个阶段, 即弹性接触, 第一阶弹塑性接触, 第 二阶弹塑性接触, 塑性接触。下面对每个阶段进行 讨论。

\subsection{1 弹性阶段}

由文献[25]Hertz 接触理论, 临界弹性接触 量为

$$
\omega_{c e}^{*}=\left(\frac{\pi K H}{2 E}\right)^{2} R=\left(\frac{\pi K \phi}{2}\right)^{2} R
$$

式中, $K=0.454+0.41 v ; H$ 为较软接触面的硬度; $E$ 为等效弹性模量; $v$ 为泊松比。对于 $R>\omega$ 时, 由 Hertz 接触理论可得接触面积和接触力为

$$
\left\{\begin{array}{l}
a=\pi R \omega \\
P_{e}(a)=\frac{4}{3} \sqrt{\pi} \cdot E \cdot G^{D-1} \cdot a^{\frac{3-D}{2}}
\end{array}\right.
$$

当微凸体变形量为 $\omega_{c e}^{*}$ 时, 由式(6)、(7)得到临界弹 性接触面积和临界弹性接触力为

$$
\left\{\begin{array}{l}
a_{c e}^{*}=\frac{1}{\pi}\left(\frac{K \phi}{2}\right)^{2}\left(\frac{L^{D}}{G^{D-1}}\right)^{2} \\
P_{c e}^{*}=\frac{4 \sqrt{\pi} E G^{D-1}}{3 L^{D}} a^{\frac{3}{2}}
\end{array}\right.
$$

\subsection{2 弹塑性阶段}

由文献[15], KOGUT 和 ETSION 采用有限元的 思想分析了球形表面与刚性平面接触产生的弹塑性 变形, 并把此过程分为两个阶段: 第一弹塑性阶段, 第二弹塑性阶段。

对于第一弹塑性阶段 $\omega_{c e}^{*}<\omega \leqslant 6 \omega_{c e}^{*}$

$$
\left\{\begin{array}{l}
a=a_{c e}^{*} \cdot 0.93\left(\frac{\omega}{\omega_{c e}^{*}}\right)^{1.136} \\
P_{e p 1}(a)=P_{c e}^{*} \cdot 1.1282\left(\frac{a}{a_{c e}^{*}}\right)^{\frac{1.425}{1.136}}
\end{array}\right.
$$

对于第二弹塑性阶段 $6 \omega_{c e}^{*}<\omega \leqslant 110 \omega_{c e}^{*}$

$$
\left\{\begin{array}{l}
a=a_{c e}^{*} \cdot 0.94\left(\frac{\omega}{\omega_{c e}^{*}}\right)^{1.146} \\
P_{e p 2}(a)=P_{c e}^{*} \cdot 1.4988\left(\frac{a}{a_{c e}^{*}}\right)^{\frac{1.263}{1.146}}
\end{array}\right.
$$

根据式(9)、(10)可得到两弹塑性临界点的临界接触 面积和临界接触压力为

$$
\left\{\begin{array}{l}
\left\{\begin{array}{l}
a_{c e p 1}^{*}=7.1197 a_{c e}^{*} \\
P_{c e p 1}^{*}=\frac{2}{3} K H \cdot 1.1282 a_{c e}^{*-0.2544} \cdot a^{1.2544}
\end{array}\right. \\
\left\{\begin{array}{l}
a_{c e p 2}^{*}=205.3827 a_{c e}^{*} \\
P_{c e p 2}^{*}=\frac{2}{3} K H \cdot 1.4988 a_{c e}^{*-0.1021} \cdot a^{1.1021}
\end{array}\right.
\end{array}\right.
$$

\subsection{3 塑性阶段}

当 $a>a_{c e p 2}^{*}$ 或 $\omega>110 \omega_{c e}^{*}$ 时发生全塑性变形, 由 文献[26-27]可知, 接触面积和接触力为

$$
\left\{\begin{array}{l}
a=2 \pi R \omega \\
P_{p}(a)=H a
\end{array}\right.
$$

综上, 由式(2) (5)可知, 尺度序数 $n$ 对取样长度 $L$, 微凸体高度 $\delta$ 和接触变形 $\omega$ 都有一定的影响。进一 步由式(6) (12) 分析得: 尺度序数 $n$ 对接触参数有 一定的影响。

\section{2 粗糙接触表面接触模型建立}

根据 WM 分形函数, 粗糙表面是由不同尺度的 微凸体累加而成。如图 3 所示, 当刚性平面产生一 定的位移时, 具有相同变形量 $\omega$ 的不同微凸体接触 状态不同，接触面接触状态与尺度序数 $n$ 有一定的 关系。

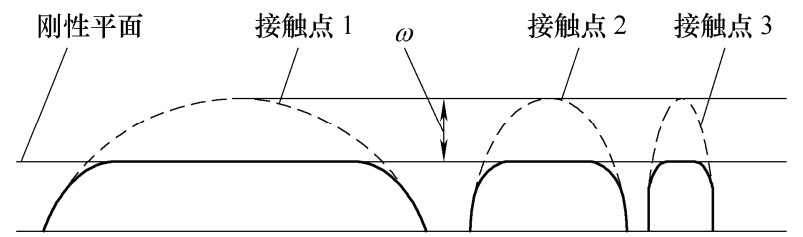

图 3 粗䊁面接触模型

在文献[23-24]中，成雨等研究了不同尺度序数 $n$ 对临界接触状态的影响, 得出结论: 6 个连续的尺 度序数对接触参数起决定性的作用, 完全可以代表 当前接触性态, 而不需要如 $\mathrm{W}-\mathrm{M}$ 函数中的尺度序 数 $n$ 要逼近于无穷。

本文建立的新模型在此基础上引入线性因子, 采用新积分求和方法对接触参数进行计算。

\subsection{1 微凸体的面积分布函数}

MANDELBORT $^{[28]} 1975$ 年发现海洋面岛屿的 面积分布遵循幂函数规律, 即 


$$
N(A>a)=a^{-\frac{D}{2}}
$$

式中, $N$ 为面积大于 $a$ 的岛屿数; 并得出自相似曲 线维数 $D$ 与表面维数 $D_{s}$ 有关, $D=D_{s}-1$ 。在此基 础上得出接触面垂直轮廓线最大接触点的数量为

$$
N(A>a)=\left(\frac{a_{L}}{a}\right)^{\frac{D}{2}}
$$

式中, $a_{L}$ 为所有接触微凸体中的最大接触面积。

由此得接触点的面积分布函数为

$$
n(a)=\left|\frac{\mathrm{d} N}{\mathrm{~d} a}\right|=\frac{D}{2} \cdot \frac{a_{L}^{\frac{D}{2}}}{a^{1+\frac{D}{2}}}
$$

在上述的基础上, 本模型采用不同的计算思路：在 轮廓线最大接触点的个数中考虑如下关系式

$$
N_{e} \subseteq N_{e p 1} \subseteq N_{e p 2} \subseteq N_{p}
$$

弹性最大接触点包含于弹塑性最大接触, 弹塑性最 大接触点包含于塑性最大接触点。先将四种接触状 态分离开, 再分析研究得到四种接触状态最大点数 目为

$$
\begin{cases}N(A>a)=\left(\frac{a_{c e}^{*}}{a}\right)^{\frac{D}{2}} & a_{L e} \leqslant a_{c e}^{*} \\ N(A>a)=\left(\frac{a_{c e p 1}^{*}}{a}\right)^{\frac{D}{2}} & a_{L e p 1} \leqslant a_{c e p 1}^{*} \\ N(A>a)=\left(\frac{a_{c e p 2}^{*}}{a}\right)^{\frac{D}{2}} & a_{L e p 2} \leqslant a_{c e p 2}^{*} \\ N(A>a)=\left(\frac{a_{L}}{a}\right)^{\frac{D}{2}} & a_{c e p 2}^{*}<a_{L}\end{cases}
$$

式中, $a_{L e}, a_{L e p 1}, a_{L e p 2}, a_{L}$, 分别为对应接触状 态下的最大接触点面积。

由式(15) (17)得到微凸体面积分布函数为

$$
\left\{\begin{array}{l}
n_{e}(a)=\frac{D}{2} \frac{a_{c e}^{* \frac{D}{2}}}{a^{1+\frac{D}{2}}} \\
n_{e p 1}(a)=\frac{D}{2} \frac{1}{a^{1+\frac{D}{2}}}\left[a_{c e p 1}^{* \frac{D}{2}}-a_{c e}^{* \frac{D}{2}}\right] \\
n_{e p 2}(a)=\frac{D}{2} \frac{1}{a^{1+\frac{D}{2}}}\left[a_{c e p 2}^{* \frac{D}{2}}-a_{c e p 1}^{*} \frac{D}{2}\right] \\
n_{p}(a)=\frac{D}{2} \frac{1}{a^{1+\frac{D}{2}}}\left[a_{L}^{\frac{D}{2}}-a_{c e p 2}^{*} \frac{D}{2}\right]
\end{array}\right.
$$

\subsection{2 接触线性因子}

当微凸体发生接触变形时, 将接触平面假想剖 分开得如图 4 所示。

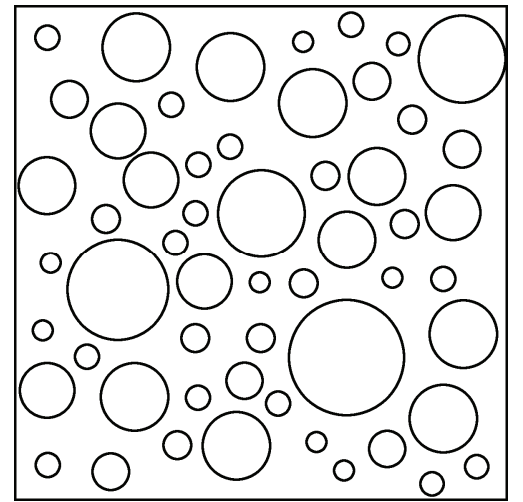

图 4 接触面假想剖分面

平面接触状态包括弹性, 弹塑性, 塑性接触状 态。接触状态随着接触量 $\omega$ 的改变也随之发生相应 的转变: 未接触的微凸体进入弹性接触状态, 弹性 接触状态转换为弹塑性接触状态, 弹塑性接触状态 转换为塑性接触状态。

微凸体在弹性接触状态时, $\omega \leqslant \delta$, 由式(4)、 (6)得出弹性接触临界尺度序数 $n_{e}$ 为

$$
n_{e}=\operatorname{ent}\left(\frac{\lg \left[(K \phi / 2)^{2} \cdot\left(\frac{1}{G}\right)^{2(D-1)}\right]}{2(D-1) \lg \gamma}\right)
$$

同理，由式(4)、(9)的分段点得弹塑性接触临界 尺度序数 $n_{e p}$ 为

$$
n_{e p}=\operatorname{ent}\left(\frac{\lg \left[6(K \phi / 2)^{2} \cdot\left(\frac{1}{G}\right)^{2(D-1)}\right]}{2(D-1) \lg \gamma}\right)
$$

同理，由式(4)、(10)的分段点得塑性接触临界 尺度序数 $n_{p}$ 为

$$
n_{p}=\operatorname{ent}\left(\frac{\lg \left[110(K \phi / 2)^{2} \cdot\left(\frac{1}{G}\right)^{2(D-1)}\right]}{2(D-1) \lg \gamma}\right)
$$

在接触过程中, 可以将接触平面的接触过程通 过引入接触系数 $K s$ 来模拟。接触量 $\omega$, 接触系数 $K s$ ，微凸体的高度 $\delta$ 满足如下关系

$$
\omega=K s \cdot \delta \quad 0 \leqslant K s \leqslant 1
$$

$K s$ 从 0 取值到 1 的物理含义为微凸体从接触产生到 完全挤压成平面。尺度序数 $n$ 影响临界接触参数 $a_{c e}^{*} 、 a_{c e p 1}^{*} 、 a_{c e p 2}^{*}$ 和最大接触点参数 $a_{n L}$ 。

新接触模型的计算流程如图 5 所示，图中第 1 行第一个小方块为接触面中的截断接触面积, 将其 按照式(16)和式(19) (21)拆分成 $a, b, d, g$ 四种接 触面积。图中第 2 行对应的小方块 $A, B, D, G$ 表 
征接触面仅考虑单一接触状态的接触面形态。通过 线性因子 $\Phi_{i}$ 修正后求和得到真实接触面积。

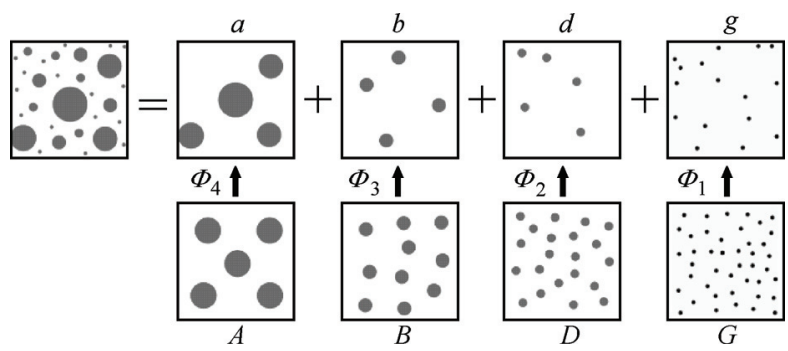

图 5 新接触模型求解方法

其中线性因子 $\Phi_{i}$ 的求解方式为: 定义真实接触 点的集合为 $M$, 理论的接触点集合为 $E$, 则有

$$
M \stackrel{T}{\longrightarrow} E
$$

其中, 式(23)表明真实点集在假设条件 $T$ 的作用下 映射到理论集 $E$ 中, $E$ 中包含了 $E_{e}$ (弹性状态集); $E_{e p 1}$ (一阶弹塑性状态集); $E_{e p 2}$ (二阶弹塑性状态集); $E_{p}$ (塑性状态集), 即

$$
\begin{cases}a_{i} \leqslant a_{c e}^{*} & \sum a_{i} \subseteq E_{e} \\ a_{c e}^{*}<a_{i} \leqslant a_{c e p 1}^{*} & \sum a_{i} \subseteq E_{e p 1} \\ a_{c e p 1}^{*}<a_{i} \leqslant a_{c e p 2}^{*} & \sum a_{i} \subseteq E_{e p 2} \\ a_{i}>a_{c e p 2}^{*} & \sum a_{i} \subseteq E_{p}\end{cases}
$$

再由不同接触状态集的测度比值得线性因子值为

$$
\left\{\begin{array}{l}
\Phi_{1}=\frac{M\left(E_{e}\right)}{M(E)} \\
\Phi_{2}=\frac{M\left(E_{e p 1}\right)}{M(E)} \\
\Phi_{3}=\frac{M\left(E_{e p 2}\right)}{M(E)} \\
\Phi_{4}=\frac{M\left(E_{p}\right)}{M(E)}
\end{array}\right.
$$

式中, 各个测度 $M\left(E_{i}\right)$ 由临界尺度序数 $n_{i}$ 和最大接 触点参数 $a_{n L}$ 给出, 即

$$
\left\{\begin{aligned}
M\left(E_{e}\right)= & \sum_{n_{\min }}^{n_{e}} a_{n L}+\sum_{n_{e}+1}^{n_{\max }} a_{n c e}^{* \frac{2-D}{2}} \cdot a_{n L}^{\frac{D}{2}} \\
M\left(E_{e p 1}\right)= & \sum_{n_{e}+1}^{n_{e p}}\left(a_{n L}^{\frac{2-D}{2}}-a_{n c e}^{* \frac{2-D}{2}}\right) \cdot a_{n L}^{\frac{D}{2}}+ \\
& \sum_{n_{e p}+1}^{n_{\max }}\left(a_{n c e p 1}^{* \frac{2-D}{2}}-a_{n c e}^{* \frac{2-D}{2}}\right) \cdot a_{n L}^{\frac{D}{2}} \\
M\left(E_{e p 2}\right)= & \sum_{n_{e p}+1}^{n_{p}}\left(a_{n L}^{\frac{2-D}{2}}-a_{n c e p 1}^{*}\right) \cdot a_{n L}^{\frac{2-D}{2}}+ \\
M\left(E_{p}\right)= & \sum_{n_{p}+1}^{n_{\max }}\left(a_{n c e p 2}^{\frac{2-D}{2}}-a_{n c e p 1}^{*} \frac{2-D}{2}\right) \cdot a_{n L}^{\frac{D}{2}} \\
n_{p}+1 & \left.a_{n L}^{\frac{2-D}{2}}-a_{n c e p 2}^{*}{ }^{\frac{2-D}{2}}\right) \cdot a_{n L}^{\frac{D}{2}}
\end{aligned}\right.
$$

式中, $M\left(E_{e}\right), M\left(E_{e p 1}\right), M\left(E_{e p 2}\right), M\left(E_{p}\right)$ 分别 是不同接触状态集的测度值, $M(E)$ 为总测度。 $a_{n c e}^{*}, a_{n c e p 1}^{*}, a_{n c e p 2}^{*}$ 和 $a_{n L}$ 为随尺度序数 $n$ 变化的接 触面临界参数和接触面最大面参数。

\subsection{3 真实接触面积和接触载荷}

粗粮表面接触时, 真实接触面积 $A_{r}$ 和真实接触 载荷 $P_{r}$ 是接触平面上所有微凸体作用的总和, 对单 个微凸体的接触参数进行积分求和得到粗敉平面的 接触参数如下。

真实接触面积

$$
A_{r}=A_{e}+A_{e p 1}+A_{e p 2}+A_{p}
$$

式中, $A_{e}$ 为弹性接触状态下的微凸体面积总和; $A_{e p 1}$ 为一阶弹塑性接触状态下的微凸体面积总和; $A_{e p 2}$ 为二阶弹塑性接触状态下的微凸体面积总和; $A_{p}$ 为 塑性接触状态下的微凸体面积总和。由式(18)、(25) 得式 $(28) \sim(31)$

$$
\begin{gathered}
A_{e}=\Phi_{1} \cdot \int_{0}^{a_{e L}} n_{e}(a) \cdot a \cdot \mathrm{d} a \\
A_{e p 1}=\Phi_{2} \cdot \int_{0}^{a_{e p 1 L}} n_{e p 1}(a) \cdot a \cdot \mathrm{d} a \\
A_{e p 2}=\Phi_{3} \cdot \int_{0}^{a_{e p 2 L}} n_{e p 2}(a) \cdot a \cdot \mathrm{d} a \\
A_{p}=\Phi_{4} \cdot \int_{0}^{a_{L}} n_{p}(a) \cdot a \cdot \mathrm{d} a
\end{gathered}
$$

真实接触载荷

$$
P_{r}=P_{e}+P_{e p 1}+P_{e p 2}+P_{p}
$$

式中, $P_{e}$ 为弹性接触状态下微凸体变形产生的接触 力总和; $P_{e p 1}$ 为一阶弹塑性接触状态下微凸体变形 产生的力总和; $P_{e p 2}$ 为二阶弹塑性接触状态下微凸 体变形产生的力总和; $P_{p}$ 为塑性接触状态下微凸体 变形产生的力总和。由式(7), (9), (10), (12), (18), (25) 得式(33) (36)

$$
\begin{gathered}
P_{\mathrm{e}}=\Phi_{1} \cdot \int_{0}^{a_{e L}} p_{e}(a) \cdot n_{e}(a) \cdot \mathrm{d} a \\
P_{e p 1}=\Phi_{2} \cdot \int_{0}^{a_{e p 1 L}} p_{e p 1}(a) \cdot n_{e p 1}(a) \cdot \mathrm{d} a \\
P_{e p 2}=\Phi_{3} \cdot \int_{0}^{a_{e p 2 L}} p_{e p 2}(a) \cdot n_{e p 2}(a) \cdot \mathrm{d} a \\
P_{p}=\Phi_{4} \cdot \int_{0}^{a_{p L}} p_{p}(a) \cdot n_{p}(a) \cdot \mathrm{d} a
\end{gathered}
$$

式(28) $(31)$ 和式(33) $(36)$ 中的 $a_{e L}, a_{e p 1 L}, a_{e p 2 L}$, $a_{p L}$ 分别为对应接触状态下的最大接触面积。将式 (28) (31) 代入式(27)并对粗糙面量纲一化得 


$$
A_{r}^{*}=\frac{A_{r}}{A_{a}}
$$

将式(33) (36)代入式(32)并对粗糙表面接触力进行 量纲一化得

$$
P_{r}^{*}=\frac{P_{r}}{E \cdot A_{a}}
$$

式中, $E$ 为弹性模量; $A_{a}$ 为名义接触面积。

对上述公式分析可以得到: 任意确定的粗粘 表面 $A_{i}$, 当产生一定的接触量时, 其弹性、第一 阶弹塑性、第二阶弹塑性和塑性临界面积随着尺 度序数 $n$ 的变化而不同。并且, 微凸体接触点临 界变形 $\omega_{c e i}^{*}$ 和 $\omega_{c p i}^{*}$ 也随着 $n$ 的变化而不同, 每种接 触状态下微凸体的最大面积 $a_{L}$ 和尺度序数 $n$ 有 关, 采用线性因子 $\Phi_{i}$ 来修正每种接触状态所占的 比例。综上, 接触面积 $A$ 与线性因子 $\Phi_{i}$ 和每种接 触状态下的最大接触点面积 $a_{n L}$ 成一定关系, 而真 实接触载荷 $P$ 的大小也取决于最大接触点面积 $a_{n L}$ 和线性因子 $\Phi_{i}$, 由此可以建立载荷与真实接触 面积的关系。

\section{3 模型的仿真验证}

新模型考虑了弹性、弹塑性、塑性接触状态, 考虑了多尺度 $n$ 下的接触参数, 分析线性因子随分 形参数 $D, G$ 的关系; 与 $\mathrm{GW}$ 模型和 $\mathrm{MB}$ 模型进行 比较; 与其他考虑尺度参数影响的接触模型进行 对比。

\section{1 线性因子与分形参数的分析}

新模型建立基于 2 维 W-M 分形函数和文献 [24]。为了便于比较, 分形维数 $D$ 取 $1.1 \sim 1.9$, 尺 寸系数 $G$ 取 $10^{-17} \sim 10^{-6} \mathrm{~m}$, 泊松比 $v=0.17$, 弹性模 量 $E=72 \times 10^{9} \mathrm{~N} / \mathrm{m}^{2}$, 硬度 $H=5.5 \times 10^{9} \mathrm{~N} / \mathrm{m}^{2}$ 。

\subsection{1 线性因子与接触系数 $K s$}

线性因子的仿真, 取 $D=1.49, G=10^{-9} \mathrm{~m}$, 得 出图 6 所示的结果, 纵坐标代表 $\Phi_{1}, \Phi_{2}, \Phi_{3}, \Phi_{4}$ 的 大小, 横坐标接触系数 $K s$ 表征了接触量的大小, $K s$ 从 0 到 1 的过程代表微凸体开始接触到整个微凸 体压扁为止。

从图 6 中可以看出: (1) 在一定 $K s$ 下, 微凸体 的弹性变形远多于一阶弹塑性变形、二阶弹塑性变 形和塑性变形; 一阶弹塑性变形多于二阶弹塑性变 形, 二阶弹塑性变形多于塑性变形。这与实际是相 符合的。因为, 当两个平面开始接触时, 由于表面 微凸体的不平整, 导致了极小一部分区域处于塑性

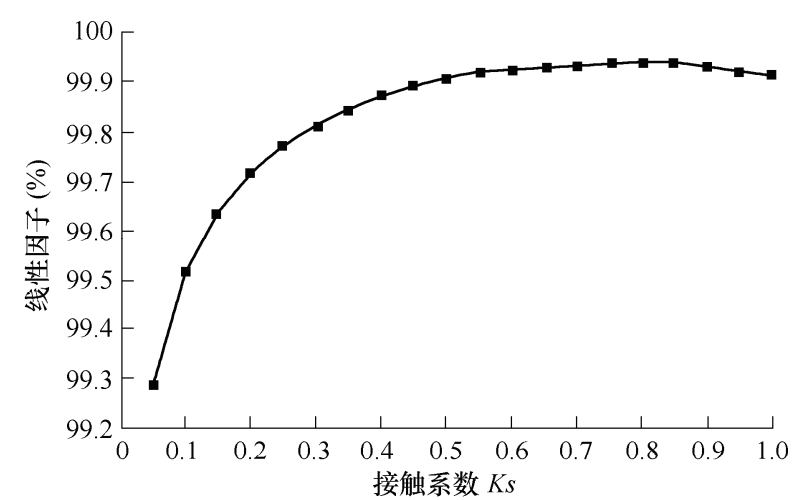

(a) 弹性接触状态

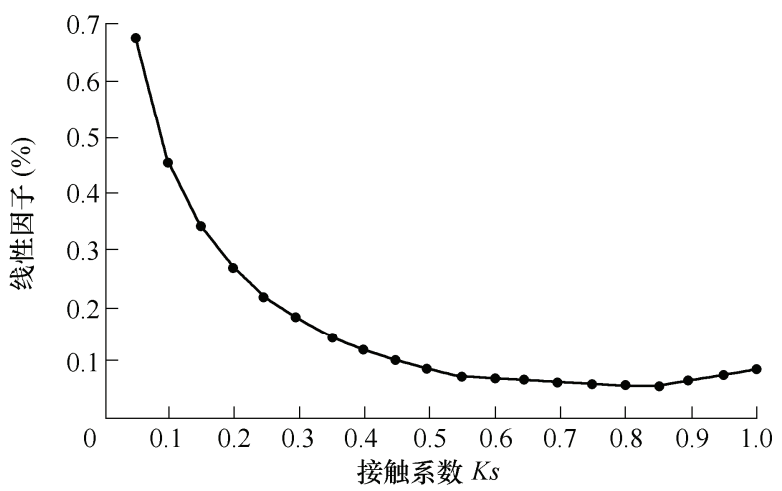

(b) 一阶弹塑性接触状态

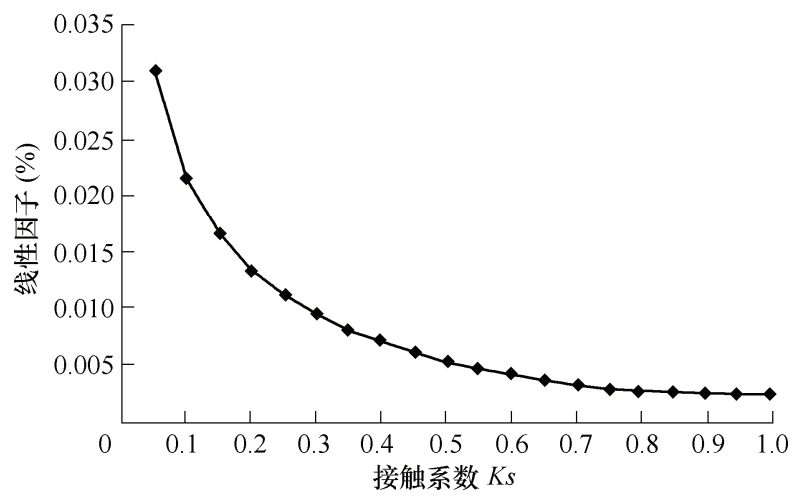

(c) 二阶弹塑性接触状态

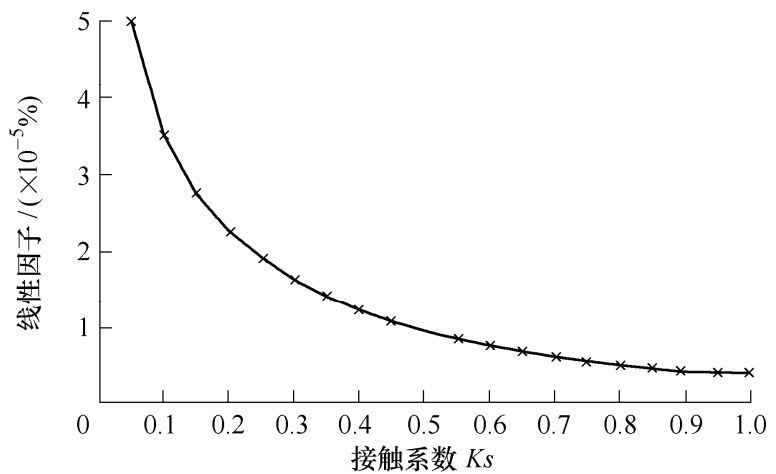

(d) 塑性接触状态

图 6 不同接触量下的线性因子百分比

变形、较小一部分区域处于二阶弹塑性变形、小部 分区域处于一阶弹塑性变形, 其产生极不均匀的局 部应力, 大部分区域处于弹性变形, 产生较为均匀 的平面应力; (2) 接触量 $K s$ 的增加, 微凸体弹性接 
触比例在 $K s<0.835$ 时处于增加状态。微凸体一阶 弹塑性、二阶弹塑性和塑性接触比例都处于下降阶 段, 这是因为, 随着接触量 $K s$ 的增加, 越来越多的 微凸体接触, 弹性接触面积呈现增加的趋势, 此时, 一阶弹塑性、二阶弹塑性和塑性比例也在增大, 但 增大速率远远小于弹性比例的增加, 因此在图 $6 \mathrm{~b}$, $6 \mathrm{c}$ 中呈现出下降的趋势; (3) 当接触系数 $K s>0.835$ 时, 微凸体弹性比例呈现下降趋势; 一阶弹塑性比 例呈现上升趋势; 而二阶弹塑性比例和塑性比例仍 处于下降趋势, 但趋势变的平缓。这是因为, 当接 触量到一定状态时, 微凸体进入接触状态的数目趋 于平稳值, 随着接触量的进一步增加, 部分弹性接 触转变为一阶弹塑性接触, 一阶弹塑性接触转变为 二阶弹塑性接触, 二阶弹塑性接触转变为塑性接触。 但是, 弹性向一阶弹塑性转变的微凸体要远多于一 阶弹塑性向二阶弹塑性转变、二阶弹塑性向塑性转 变的微凸体, 因此在图 $6 \mathrm{c}$ 中, $K s>0.835$ 时, 二阶 弹塑性、塑性接触比例仍然缓慢下降。其原因来源 于两部分, 第一, 处于后面接触状态的微凸体占总 接触微凸体数的比例非常少, 使得其影响能力较 弱。第二，接触状态较前的微凸体向接触状态较后 的微凸体转变更容易 ${ }^{[26]}$ 。

\section{1 .2 线性因子与分形参数 $D$}

如图 7 所示 (取 $G=10^{-9} \mathrm{~m}$ ), (1) 弹性接触状态, 当 $D<1.5$ 时, 随着分形参数 $D$ 的增加, 线性因子 $\Phi_{1}$, $\Phi_{2}, \Phi_{3}, \Phi_{4}$ 逐步增加; 当 $D>1.5$ 时, $D$ 的变化对 线性因子的影响趋于平缓; (2)一阶弹塑性接触状 态, 当 $D<1.5$ 时, $D$ 对线性因子的影响较大, 当 $D>1.5$ 时, $D$ 对线性因子的影响趋于平缓; (3) 二 阶弹塑性接触状态和塑性接触状态, 当 $D<1.2$ 和 $D>1.5$ 时, $D$ 对线性因子的影响较小，当 $1.2<D<$ 1.5 时, $D$ 对线性因子的影响较大; (4) 综上, $D$ 对 线性因子前两种接触状态影响较大, 对后两种接触 状态影响较小。并且, 接触系数 $K s$ 对后三种接触状 态的线性因子影响较小。

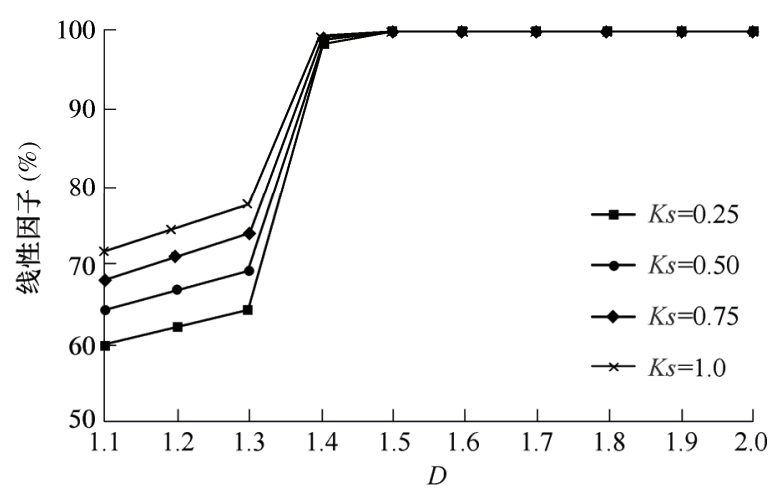

(a) 弹性接触状态

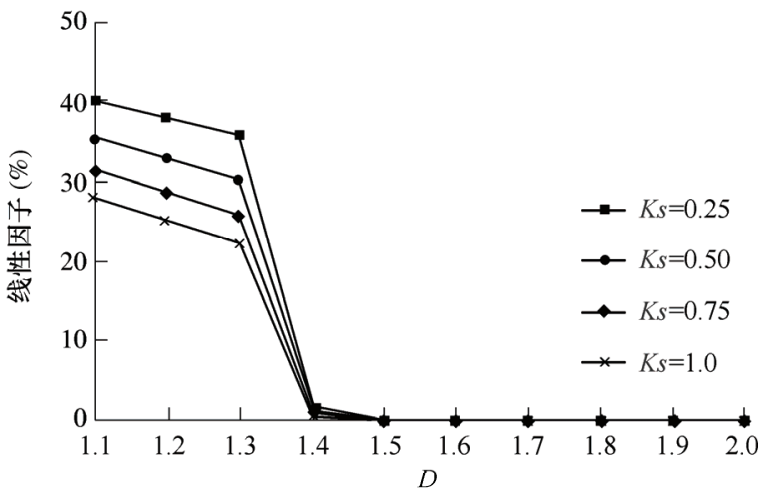

(b) 一阶弹塑性接触状态

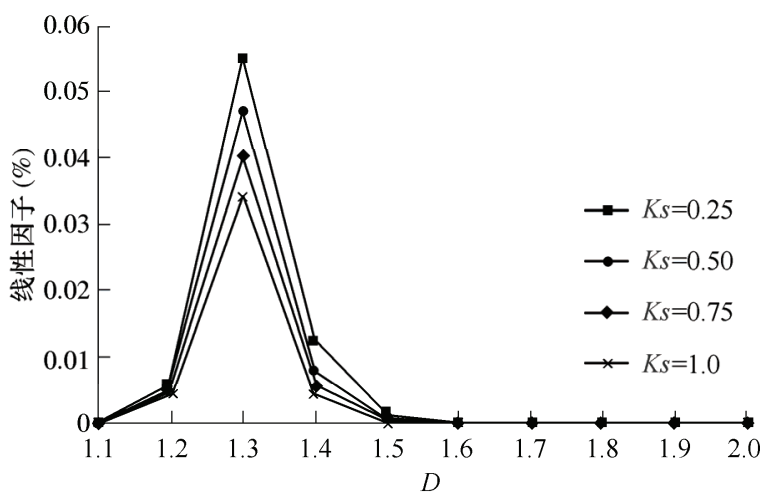

(c) 二阶弹塑性接触状态

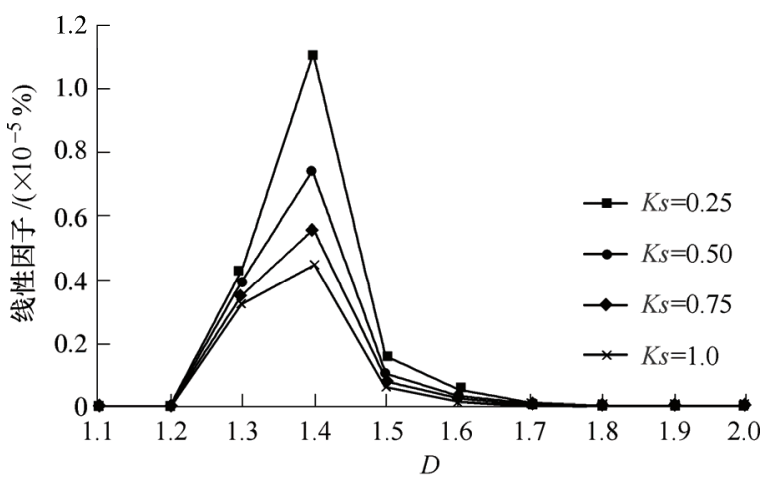

(d) 塑性接触状态

图 7 线性因子与 $D$ 的关系

分析：对于不同的接触系数 $K s$, 分形维数 $D$ 对 线性因子 $\Phi_{1}, \Phi_{2}, \Phi_{3}, \Phi_{4}$ 的影响趋势都相同。对 于某特定的接触系数 $K s$, 随着 $D$ 的增加, W-M 分 形函数在横轴尺度上细化, 待研究微凸体的数量增 加, 由分析可知微凸体弹性状态的数目会迅速增加, 并且其总量大于一阶弹塑性微凸体总量。由于后两 种接触状态的微凸体数目总体较少, 虽然分形维数 $D$ 对其线性因子有一定的影响，但相对于前两种接 触状态的影响较弱。分形维数 $D$ 较大时, 由于线性 因子是基于多尺度 $n$ 的条件下建立的。因此， $D$ 对 线性因子的影响较小。

\section{1 .3 线性因子与特征系数 $G$}

由第 3.1.2 节的仿真分析可以看出, 线性因子随 分形维数 $D$ 变化时, 会在某些点出现突变跳跃现象。 
经分析研究发现, 随着特征系数 $G$ 的变化, 突变区 域会发生变化, 取 $G$ 为 $2.5 \times 10^{-20} \mathrm{~m}$ 至 $2.5 \times 10^{-6} \mathrm{~m}$, 得到关系曲线如图 8 所示, 图中横坐标代表了特征 系数 $G$ 取对数的值, 纵坐标代表了不同的特征系数 $G$ 所对应第 3.1.2 节中的突变区域。(1) 从散点图中 可以看出, 弹性和一阶弹塑性对应的突变区域分形 维数 $D$ 偏差不大, 均低于二阶弹塑性和塑性接触状 态; (2) 从散点图拟合的线性趋势上可得：随着 $G$ 的增大, 线性因子百分比对应的突变区域随分形维 数 $D$ 呈增大趋势。

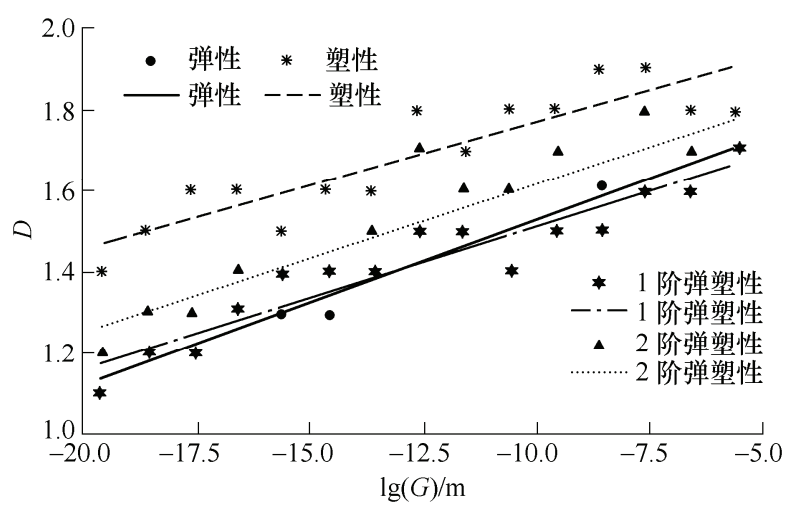

图 8 突变区域与 $D$ 和 $G$ 的关系曲线

分析: 特征系数 $G$ 表征了轮廓曲线上微凸体纵向 高度的大小, 其值越大表明轮廓线越粗粞。分形维数 $D$ 表征了轮廓线的精细程度, 在一定范围内, $D$ 越大 表明轮廓线的精细程度越好。特征系数 $G$ 较小时, 接 触面变得 “平整”, 这时研究粗糙接触表面的接触特 性就需要较大的分形维数 $D$, 随着 $D$ 的增大才能将更 多微凸体接触特性考虑进去, 进而得出合理的结果。 因此, 如图 8 所示曲线关系, 随着特征系数 $G$ 的增加, 突变不稳定区域对应的分形维数 $D$ 也随之增加。

\section{2 粗䊁面接触模型的验证}

将考虑多尺度序数 $n$ 的新模型与经典模型作对 比分析，进一步验证模型的可行性。

\subsection{1 新模型与 $\mathrm{MB}$ 模型和 $\mathrm{GW}$ 模型对比}

现把新模型与经典的 $\mathrm{GW}$ 模型 ${ }^{[5]}$ 和 $\mathrm{MB}$ 模型 ${ }^{[14]}$ 以及 BHUSHAN 试验数据 ${ }^{[29]}$ 在同一条件下作对比。 取 $D=1.49, Y / E=0.05, G=10^{-16} \mathrm{~m}$, 得出图 9 所示 的关系曲线。

(1) 当接触力较小时, $F_{r}^{*}<2.8$ 时, 新模型与经 典的 MB 模型比较相近, 在相同接触力的条件下, 接触面积要大于 MB 模型, 并且与 BHUSHAN 的试 验数据吻合相对 MB 模型好一些。这是合理的, 因 为 $\mathrm{MB}$ 模型忽略了弹塑性阶段的影响, 而新模型考 虑了多尺度下微凸体的 4 种接触性态, 因此, 新模 型得出的接触面积要比 $\mathrm{MB}$ 模型的大一些, 比经典
的 MB 模型具有更高的精度。

（2）在接触载荷较大时, 即 $F_{r}^{*}>2.8$ 时, 新模型 与试验数据的偏差越来越小, 在 $5<F_{r}^{*}<7$ 时, 与试 验数据几近重合, 更加精确地表征出了接触面积与 接触力的关系。

(3) 在相同接触力下, 新模型得到的接触面积 较 $\mathrm{GW}$ 模型预测结果更大一些, 这是因为 $\mathrm{GW}$ 模型 是基于统计学得出的模型, 其数据精度受制于仪器 设备的采样精度, 不能完全真实地反映出接触面的 接触特性, 并且它的建立仅考虑了单一尺度, 因此 在精度上欠缺一些。

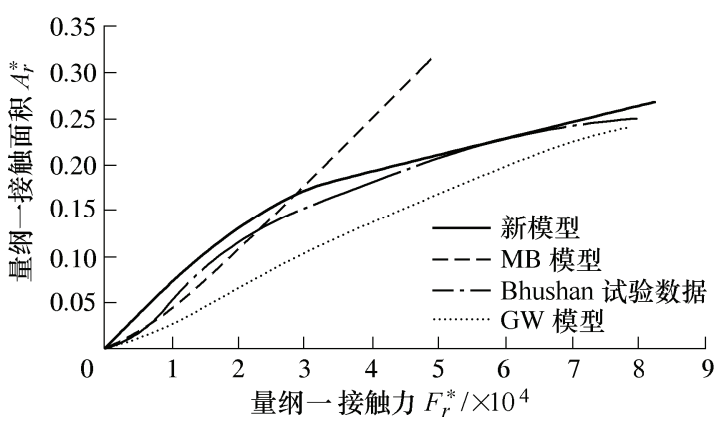

图 9 新模型与 $\mathrm{GW}$ 模型和 $\mathrm{MB}$ 模型的对比

\subsection{2 新模型与现有考虑尺度的模型进行对比}

将新模型与成雨模型 ${ }^{[23]}$ 进行对比，在第 3.2.1 节的条件下, 如图 10 所示, 两个模型在总体趋势和 数值上很接近, 但是随着接触力的增加, 接触面积 存在一定的误差, 并且随接触力的增大误差增大。 这是因为成雨在接触模型的建立时仅考虑了不同尺 度 $n$ 对临界参数的影响, 再由临界参数得出接触面 的接触参数, 而没有对大尺度范围内做宏观定量分 析。而本文在建立模型时考虑了大尺度范围内的影 响, 通过线性因子和新的积分求和方法进行接触参 数的计算。因此, 在结果方面相对于成雨模型更加 接近实际状况, 新模型与试验结果的误差更小。

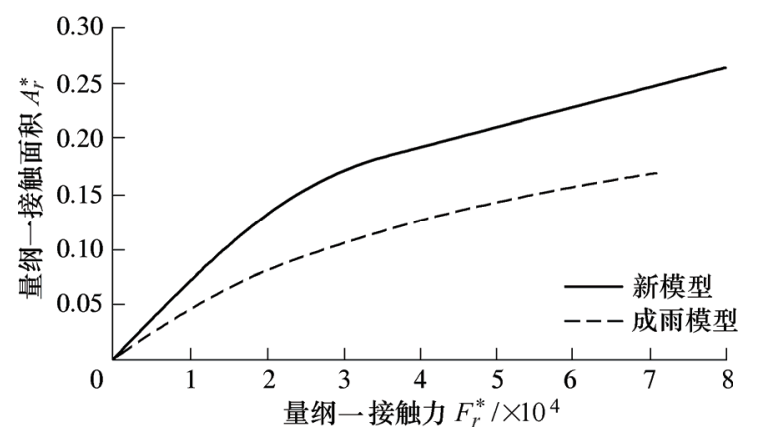

图 10 新模型与成雨模型的对比

\section{4 结论}

（1）不同尺度的接触参数对接触面性态有不同 
的影响。考虑不同尺度的影响, 在经典接触模型和 Hertz 接触理论的基础上建立新的接触模型。新模型 采用新的计算方法与流程, 考虑多尺度的影响, 引 入线性因子和新的分布函数对接触参数进行求解, 其预测结果在精度上有所提高。相比经典的接触模 型在小载荷和大载荷作用下都具有较高的精度。

（2）考虑多尺度特性得到线性因子 $\Phi_{i}$ 对接触参 数修正。线性因子预测结果与经典接触理论结果相 同, 微凸体的变形顺序为: 弹性变形, 一阶弹塑性 变形, 二阶弹塑性变形, 塑性变形。

（3）线性因子的取值与尺度序数 $n$ 、分形维数 $D$ 和特征系数 $G$ 有关; 计算模型时应选取合适的 $D$, $G$ 参数。引入线性因子的新接触模型相比其他接触 模型在相同条件下, 预测结果趋势一致, 且在精度 方面有进一步的提高。

（4）虽然新模型的计算精度有所提高, 但对特 征系数 $\mathrm{G}$ 引起的突变效应仍未有定性研究, 可以成 为下一步研究方向。通过对新模型的建立, 希望能 在工程应用中起一定的指导作用。

\section{参 考 文 献}

[1] 陈奇, 黄守武, 张振, 等. 考虑摩擦因素的两圆柱体表 面接触承载能力的分形模型研究 [J]. 机械工程学报, 2016, 52(7): 114-121.

CHEN Qi, HUANG Shouwu, ZHANG Zhen, et al. Research on fractal contact model for contact carrying capacity of two cylinder's surfaces considering friction factors[J]. Journal of Mechanical Engineering, 2016, 52(7): 114-121.

[2] 魏龙, 张鹏高, 刘其和, 等. 接触式机械密封端面摩擦 系数影响因素分析与试验 [J]. 摩擦学学报, 2016,36(3): 354-361.

WEI Long, ZHANG Penggao, LIU Qihe, et al. Influencing factors analysis and experiments of friction coefficient between the end faces for contact mechanical seals[J]. Tribology, 2016, 36(3): 354-361.

[3] 嵇正波, 孙见君, 陆建花, 等. 基于逾渗理论的机械密 封界面静态泄漏预测方法[J]. 摩擦学学报, 2017,37(6): 734-742.

JI Zhengbo， SUN Jianjun， LU Jianhua， et al. Predicting method for static leakage of contacting mechanical seals interface based on percolation theory[J]. Tribology, 2017, 37(6): $734-742$.

[4] 田小龙, 王雯, 傅卫平, 等. 考虑微凸体相互作用的机 械结合面接触刚度模型 [J]. 机械工程学报, 2017, 53(17): 149-159.

TIAN Xiaolong, WANG Wen, FU Weiping, et al. Contact stiffness model of mechanical joint surfaces considering the asperity interactions[J]. Journal of Mechanical Engineering, 2017, 53(17): 149-159.

[5] GREENWOOD J A, WILLIAMSON J B P. Contact of nominally flat surfaces[J]. Proceedings of the Royal Society of London, 1966, 295(1442): 300-319.

[6] WHITEHOUSE D J, ARCHARD J F. The properties of random surfaces of significance in their contact[J]. Proc. Roy. Soc. London, 1970, 316(1524): 97-121.

[7] NAYAK P R. Random process model of rough surfaces in plastic contact[J]. Wear, 1971, 26(3): 398.

[8] BUSH A W, GIBSON R D, THOMAS T R. The elastic contact of a rough surface[J]. Wear, 1975, 35(1): 87-111.

[9] CIAVARELlA M, GREENWOOD J A, PAGGI M. Inclusion of "interaction" in the Greenwood and Williamson contact theory[J]. Wear, 2008, 265(5-6): 729-734.

[10] ZHAO Y, MAIETTA D M, CHANG L. An asperity microcontact model incorporating the transition from elastic deformation to fully plastic flow[J]. Journal of Tribology, 2000, 122(1): 86-93.

[11] ZHAO Y, CHANG L. A Model of asperity interactions in elastic-plastic contact of rough surfaces[J]. Journal of Tribology, 2001, 123(4): 857.

[12] 赵永武, 吕彦明, 蒋建忠. 新的粗粘表面弹塑性接触模 型[J]. 机械工程学报，2007，43(3): 95-101.

ZHAO Yongwu, LÜ Yanming, JIANG Jianzhong. New elastic-plastic model for the contact of rough surfaces[J]. Chinese Journal of Mechanical Engineering, 2007, 43(3): 95-101

[13] CHANG W R. An elastic-plastic model for the contact of rough surfaces[J]. Journal of Tribology，1987，109(2): 257-263.

[14] MAJUMDAR A, BHUSHAN B. Fractal model of elastic-plastic contact between rough surfaces[J]. Journal of Tribology, 1991, 113(1): 1-11.

[15] KOGUT L, ETSION I. Elastic-Plastic contact analysis of a sphere and a rigid flat[J]. Journal of Applied Mechanics, 2002, 69(5): 657-662.

[16] MORAG Y, ETSION I. Resolving the contradiction of asperities plastic to elastic mode transition in current contact models of fractal rough surfaces[J]. Wear, 2007, 262(5-6): 624-629.

[17] JACKSON R L, STREATOR J L. A multi-scale model for contact between rough surfaces[J]. Wear, 2006, 261(11-12): 1337-1347.

[18] WRIGGERS P, NETTINGSMEIER J. Homogenization and multi-scale approaches for contact problems[M]. Vienna: Springer, 2008.

[19] 孙伟, 黄信, 孙志勇, 等. 实际结合面的法向接触刚度 
多尺度计算方法 $[\mathrm{J}]$. 机械设计与制造，2017(10)：1-4. SUN Wei, HUANG Xin, SUN Zhiyong, et al. A multiscale calculation method of normal contact stiffness of actual interfaces[J]. Machinery Design and Manufacture, 2017(10): 1-4.

[20］史建成, 刘检华, 丁晓宇, 等. 基于确定性模型的金属 表面多尺度接触行为研究 [J]. 机械工程学报, 2017, 53(3): 111-120.

SHI Jianchen, LIU Jianhua, DING Xiaoyu, et al. On the multi-scale contact behavior of metal rough surface based on deterministic model[J]. Journal of Mechanical Engineering, 2017, 53(3): 111-120.

[21] MIAO X, HUANG X. A complete contact model of a fractal rough surface[J]. Wear, 2014, 309(1-2): 146-151.

[22] 丁雪兴, 严如奇, 贾永否. 基于基底长度的粗䊁表面分 形接触模型的构建与分析 [J]. 摩擦学学报, 2014,34(4): 341-347.

DING Xuexing, YAN Ruqi, JIA Yonglei. Construction and analysis of fractal contact mechanics model for rough surface based on base length[J]. Tribology, 2014, 34(4): 341-347.

[23] 成雨, 原园, 甘立, 等. 尺度相关的分形粗䊁表面弹塑 性接触力学模型 $[\mathrm{J}]$. 西北工业大学学报, 2016, 34(3): 485-492.

CHENG Yu, YUAN Yuan, GAN Li, et al. The elastic-plastic contact mechanics model related scale of rough surface[J]. Journal of Northwestern Polytechnical University, 2016, 34(3): 485-492.

[24] YUAN Y, CHENG Y, LIU K, et al. A revised Majumdar and Bhushan model of elastoplastic contact between rough surfaces[J]. Applied Surface Science, 2017, 425-432.

[25] 约翰逊 K L. 接触力学 [M]. 北京: 高等教育出版社, 1992.

JOHNSON K L. Contact mechanics [M]. Beijing: Higher Education Press, 1992.

[26] TABOR D. The hardness of metals[J]. Measurement Techniques, 1951, 5(4): 281.

[27] KOMVOPOULOS K, YAN W. A fractal analysis of stiction in microelectromechanical systems[J]. Journal of Tribology, 1997, 119(3): 391-400.

[28] MANDELBROT B B, WHEELER J A. The fractal geometry of nature[J]. American Journal of Physics, 1983, 51(4): 468.

[29] BHARAT B. The real area of contact in polymeric magnetic media-part II: Experimental data and analysis[J]. ASLE Transactions, 1985, 28(2): 181-197.

作者简介: 运睿德, 男, 1995 年出生。主要研究方向为接触面磨损和航 空发动机结构强度可靠性。

E-mail: yunruide@buaa.edu.cn 